\title{
XVIII.
}

Arbeiten aus dem Laboratorium für experimentelle Pharmakologie zu Strassburg.

\section{Ueber das Pelletierin.}

\author{
Von \\ W. v. Schroeder, \\ Privatdocent und 1. Assistent am pharmakologischen Institut zu Strassburg.
}

Die anthelminthische Wirkung der Granatwurzelrinde war sehon Plinius und Dioscorides bekannt. Später gerieth sie in Europa in Vergessenheit und im Mittelalter wurde nur der Saft der Früchte von Punica Granatum zur Bandwurmeur benutzt. Erst im Anfang dieses Jahrhunderts traten $\mathrm{Bu}$ ch an an (1805) und andere ostindische Aerzte in England, Go mez (1822) in Portugal und durch Letzteren angeregt Mérat in Frankreich wieder fuir den therapeutischen Werth der Granatwurzelrinde ein. In Deutsehland war es Schmidtmitller, welcher das Mittel wieder zu Ansehen brachte, und so wurde es 1846 in die Pharmacopoea Borussica aufgenommen. In der ersten Ausgabe der Pharmacopoea Germanica befand sich ebenfalls Cortex radicis Granati. Die Editio altera der Pharmacopoea Germanica hat an Stelle der Cortex radicis Granati einfach Cortex Granati gesetzt. Hierdurch ist auch die Stammrinde officinell geworden, denn es ist unter Cortex Granati Wurzel- oder Stammrinde oder ein Gemenge beider zu verstehen. Was die Bestandtheile der Granatrinde anbetrifft, so war es lange bekannt, dass dieselbe sehr reich an Gerbsäure ist, wie schon ihr stark adstringirender Geschmack vermuthen liess. Nach Wacken roder enthält die Granatwurzelrinde 22 Proc., nach Ishikawa 20 Proc., nach Hager 25 Proc. Gerbsäure. Ausserdem wurde Mannit in ihr nachgewiesen und die Anwesenheit eines Alkaloids, Punicin oder Granatin genannt, vermuthet. Doch erst Tanret ${ }^{1}$ ) gelang es 1878 Alkaloide aus der Granatrinde zu isoliren. Er unterscheidet 4 in derselben, die in chemischer Beziehung einander ziemlich nahe stehen.

1) Bullet. de la soc. chim. Bd. 32. p. 464; Bd. 36 . p. 256. 


$$
\begin{aligned}
& \text { Pelletierin - } \mathrm{C}_{8} \mathrm{H}_{13} \mathrm{NO} \\
& \text { Isopelletierin - } \mathrm{C}_{8} \mathrm{H}_{15} \mathrm{NO} \\
& \text { Pseudopelletierin - } \mathrm{C}_{9} \mathrm{H}_{15} \mathrm{NO} \\
& \text { Methylpelletierin - } \mathrm{C}_{9} \mathrm{H}_{17} \mathrm{NO} .
\end{aligned}
$$

Alle diese Basen sind flichtig und bilden gut krystallisirende Salze. - Bei dem höchst widerwärtigen Geschmack des Macerationsdecocts der Granatwurzelrinde und den mancherlei Unzuträglichkeiten, welche die Benutzung desselben bei der Bandwurmcur mit sich bringt, musste es von grösstem therapeutischen Interesse sein, festzustellen, ob die Granatwurzelrinde in der That ihrem Gehalt an oben genanntèn Alkaloiden ibre anthelminthische Wirkung verdankt. Mit grossem Eifer wurden von versehiedenen französischen Aerzten Versuche in dieser Richtung angestellt, auf deren Besprechung ich später eingehen werde, welche zu dem Resultate fuhrten, dass man mit den Salzen des Pelletierins und Isopelletierins allein auch im Stande ist, die Bandwurmeur erfolgreich auszuftihren. Die Salze des Pseudo- und Methylpelletierins erwiesen sich, in den angewandten Dosen wenigstens, ohne anthelminthischen Effect, während sie in physiologischer Beziehung qualitativ die gleiche Wirkung zeigten wie das Pelletierin und Isopelletierin, aber nur bedeutend weniger toxisch waren, wie letztere Basen. Es musste also die Entdeckung Tanret's, durch welche an Stelle eines widerwärtig schmeckenden Decocts ein reines Salz gesetzt werden konnte, als wesentlicher Fortschritt bezeichnet werden. - In Nachfolgendem habe ich die physiologische Wirkung des Pelletierins einer experimentellen Untersuchung unterzogen. Musste doch die Frage beantwortet werden, ob die Pelletierine als besondere pharmakologische Gruppe anzusehen sind, oder einer der bekannten als Glieder eingefügt werden können, wie denn französische Autoren das Pelletierin den curareartig wịrkenden Giften beizählen. Zu meinen Versuchen habe ich das krystallisirte salzsaure und schwefelsaure Salz des Pelletierin benutzt, das ich der Freundlichkeit von Herrn Merck verdankte. Dasselbe war vom Methyl- und Pseudopelletierin nach den von Tanret gegebenen Vorschriften befreit worden.

\section{Wirkung des Pelletierin auf Frösche.}

Injicirt man Fröschen subcutan $0,01-0,02 \mathrm{~g}$ eines Pelletierinsalzes, so beobachtet man nach etwa 10-30 Minuten eine deutliche Zunahme der Reflexerregbarkeit. Der Zustand der gesteigerten Reflexerregbarkeit erreicht sehr allmählich, am 2. oder 3. Tag sein Maximum, um dann nach ca. 5-6 Tagen langsam zur Norm wieder zurück- 
zukehren. Bei maximaler Erregbarkeit treten in der Regel Tetanusanfälle ein, doch meist nur während kurzer Zeit. Die Erscheinung beruht, wie die analoge, durch Strychnin oder Morphin hervorgerufene in einer Erhöhung der spinalen Reflexe, denn sie lässt sìch ebenso an Thieren beobachten, welchen durch entsprechenden Schnitt das Gehirn vom Rückenmark getrennt ist. Man überzeugt sich ferner leicht, dass die beobachteten Tetanusanfälle, ebenso wie die durch Strychnin und Morphin erzeugten, als Reflextetani aufzufassen sind, und auch hier geht mit der abnorm gesteigerten Reflexerregbarkeit eine abnorm gesteigerte Ersehöpfbarkeit des Rückenmarks Hand in Hand. Ausser diesem Einfluss, welchen das Pelletierin auf das Rückenmark ausibt, zeigt sich, allerdings nur kurze Zeit andauernd, eine Wirkung auf das Gehirn, die in einer Lähmung besteht. In der ersten Stunde der Vergiftung, wenn bereits die Zunahme der Reflexerregbarkeit des Rückenmarkes deutlich hervorgetreten ist, benutzt der Frosch die ihm gebotene Gelegenheit zur Flucht nicht mehr, führt aber auf einen Reiz hin den Sprung in normaler Weise aus. Ja, man kann ihn, wenn man vorsichtig verfährt, auf den Rücken legen, in welcher Lage er verharrt, bis ein stärkerer Reiz ihn wieder zur Einnahme der normalen hockenden Stellung bringt.

$\mathrm{Zu}$ diesen das Centralorgan treffenden Wirkungen des Pelletierin tritt eine andere hinzu, welche, bei grösseren Dosen besonders, die Wahrnehmung der ersteren stören kann - es ist dies der Einfluss, den das Pelletierin auf den Muskel übt. Am besten lässt sich diese Erscheinung nach den obigen kleinen Dosen beobachten, wobei sie etwas später als die Zunahme der Reflexerregbarkeit des Rückenmarkes auftritt. Nöthigt man das Thier durch einen Reiz zu- einem Sprung, so bleibt es nach Ausführung desselben eine Weile mit gestreckten hinteren Extremitäten liegen und ziebt dieselben nicht wie normal momentan an. Es verhält sich das Thier also ähnlich, wie wenn es mit Veratrin vergiftet worden wäre, nur dass nach Pelletierin die Zeit, während welcher die Hinterextremitäten gestreckt bleiben, verhältnissmässig kurz ist gegen die nach Veratrin beobachtete. Es erscheint sehr wahrscheinlich, dass der Zustand des Muskels sich durch Pelletierin in demselben Sinne ändert, wie durch Veratrin; die Verkürzung erfolgt in normaler Weise, die Wiederausdehnung aber abnorm langsam. Die Muskelwirkung pflegt einen Tag anzuhalten, um dann zu verschwinden.

Dass es sich hierbei um eine Muskelwirkung handelt, macht folgender Versuch wahrscheinlich. Schaltet man ein Bein durch Unterbindung der Gefässe aus dem Kreislauf aus und führt dann die Vergiftung aus, 
so sieht man beim Sprunge das ausgeschaltene Bein normal schnell angezogen worden, während das vergiftete länger gestreckt bleibt. Es kann die Erscheinung durch Aenderungen im Zustand des Centralorgans nicht begruindet sein, sie muss eine peripher gelegene Ursache haben. Die Annahme einer curareartigen Wirkung ist nicht zulässig, denn benutzt man etwas grössere Dosen, so geht die directe Muskelerregbarkeit im vergifteten Bein schnell herab. Der ganze Charakter der Erscheinung entspricht nicht einer solchen Erklärung. Wenn die Vergiftung so weit vorgeschritten ist, dass Reflextetani eintreten, nehmen beide Beine in gleichem Grade an den Krämpfen Theil. Dann aber ist die Muskelwirkung längst schon vorliber.

Wählt man grosse Dosen $(0,04-0,06 \mathrm{~g})$, so tritt anfangs eine Zunahme der Reflexerregbarkeit ein, bald aber macht sich ein $\mathrm{Zu}$ stand von Lähmung geltend und die Respiration steht still. Die Lähmung ist theils durch die Einwirkung auf die Muskeln bedingt, denn die directe Erregbarkeit derselben ist stark herabgegangen, theils aber auch durch eine curareartige Wirkung des Pelletierins. Doch ist zu berücksichtigen, dass die curareartige Wirkung des Pelletierius nur bei Benutzung grosser Dosen oder bei Anwendung kleinerer gegen das Ende hin erst auftritt, so dass auf dieselbe weiter kein Gewicht zu legen ist. Jedenfalls ist es unrichtig, wie es $\mathrm{Du}$ jardin-Beaumetz) gethan, das Pelletierin mit dem Curare in eine Gruppe zu stellen.

Wirkung auf das Froschherz.

Durch das Pelletierin sinkt die Anzahl der Pulse. Die Erscheinung ist unabhängig vom Vagus, denn dieselbe tritt ebenso am atropinisirten Thier ein.

1. Versuch. Temporaria.

\begin{tabular}{|c|c|c|c|}
\hline \multicolumn{2}{|c|}{ Zeit } & $\begin{array}{l}\text { Pulse in } \\
1 \text { Minute }\end{array}$ & Bemerkungen \\
\hline 11 & 34 & 42 & \\
\hline & 52 & 42 & \\
\hline 12 & 18 & 43 & \\
\hline & 22 & - & $0,03 \mathrm{~g}$ salzsaures Pelletieri \\
\hline & 29 & 43 & \\
\hline & 35 & 41 & \\
\hline & 39 & 34 & \\
\hline & 42 & 30 & \\
\hline & 46 & 28 & \\
\hline & 52 & 26 & \\
\hline 1 & 30 & 27 & beendet \\
\hline
\end{tabular}

1) Bullet. de thérapeut. Bd. 98. p. 433. 
2. Versuch. Temporaria.

\begin{tabular}{|c|c|c|c|}
\hline $\begin{array}{l}\mathrm{Ze} \\
\mathrm{h}\end{array}$ & & $\begin{array}{l}\text { Pulse in } \\
1 \text { Minute }\end{array}$ & Bemerkungen \\
\hline 11 & $\begin{array}{l}24 \\
34\end{array}$ & $\begin{array}{l}37 \\
37\end{array}$ & $1 \mathrm{mg}$ Atropin \\
\hline & $\begin{array}{l}34 \\
45\end{array}$ & $\frac{37}{-}$ & $0,04 \mathrm{~g}$ salzsaures Pelletierin \\
\hline & 58 & 36 & \\
\hline 12 & 2 & 34 & \\
\hline & 17 & 23 & \\
\hline & 22 & 22 & \\
\hline & 58 & 22 & beendet \\
\hline
\end{tabular}

Der Vagus ist, wie ersichtlich, an der Verlangsamung des Pulses nicht betheiligt. Er wird im Gegentheil sehr bald gelähmt, denn in einem Zustand, wo die Ernährung des Herzens jedenfalls noch eine genügende ist, verursacht weder directe Vagusreizung Stillstand, noch ist Application von Nicotin anf die Schlagzahl des Herzens von irgend welchem Einfluss.

Da das Pelletierin veratrinähnlich auf den Muskel zu wirken scheint, so untersuchte ich seinen Einfluss auf das isolirte Froschberz am William s'schen Apparat. Bekanntlich wird durch Veratrin der Druck, den das Herz ausübt, gesteigert, da es, ebenso wie die Körper der Digitalingruppe, den Herzmuskel zu kräftigeren Contractionen veranlasst.

3. Versuch.

\begin{tabular}{|c|c|c|c|c|}
\hline \multicolumn{2}{|c|}{ Zeit } & $\begin{array}{c}\text { Pulse in } \\
1 \text { Minute }\end{array}$ & $\begin{array}{l}\text { Höhe des } \\
\text { Druckes } \\
\text { in mm Bg. }\end{array}$ & Bemerkungen \\
\hline 4 & 40 & 36 & 26 & \\
\hline & 42 & — & $\longrightarrow$ & Salzsaures Pelletierin dem Blute zugesetzt \\
\hline & 45 & 36 & 26 & \\
\hline & 50 & - & - & Pellet. zugesetzt \\
\hline & 55 & 36 & 29 & \\
\hline & 59 & 36 & 30 & $=$ \\
\hline 5 & 0 & 30 & 34 & $=$ \\
\hline & 3 & 24 & 38 & \\
\hline & 5 & 27 & 29 & $=$ \\
\hline & 6 & 30 & 30 & \\
\hline & 7 & 36 & 32 & \\
\hline & 8 & 36 & 32 & $=$ \\
\hline & 10 & 30 & 31 & \\
\hline & 12 & - & 29 & \\
\hline & 14 & 30 & 25 & $=$ \\
\hline & 15 & 30 & 23 & \\
\hline & 18 & 30 & 22 & $=$ \\
\hline & 24 & 27 & 23 & $=$ \\
\hline & 32 & 30 & 25 & \\
\hline
\end{tabular}


4. Versuch.

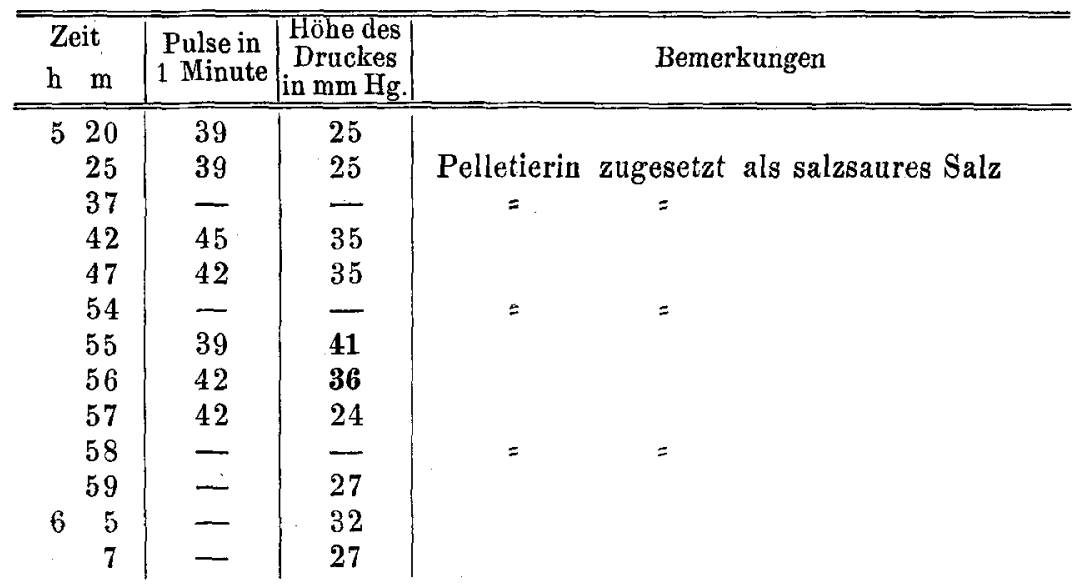

In der That steigt, wie erwartet, der Blutdruck, aber im Gegensatz zu der Wirkung des Veratrins immer nur für sehr kurze Zeiten, um dann bald wieder auf die Norm herabzusinken. Wenn also auch qualitativ das Pelletierin in seiner Wirkung mit dem Veratrin iubereinstimmt, so ist doch der quantitative Unterschied ein sehr bedeutender, denn das Pelletierin wirkt mehr wie eine den Muskel nur fur kurze Zeit erregende Substanz.

Ruft man durch Vergiftung mit Muscarin Stillstand des Froschherzens hervor, so ist man mit Pelletierin im Stande, denselben wieder aufzuheben. $\mathrm{Ob}$ in einem solchen Fall die wieder eintretende Herzbewegung durch Lähmung der Vagusendigungen nach Art des Atropins bedingt ist, oder durch Beeinflussung des Herzmuskels verursacht worden, denn auch Physostigmin und Veratrìn heben den durch Muscarin bewirkten Herzstillstand auf, das entscheidet man bekanntlich dadurch, dass man an dem wieder schlagenden Herzen zwischen Ventrikel und Vorhof eine Ligatur anlegt. War atropinartige Wirkung der Grund der wieder eingetretenen Herzbewegung, so tritt nach Anlegung der Ligatur in einigen Minuten wieder Stillstand ein. Im Fall der Wiederbeginn des Herzschlages aber durch Beeinflussung des Herzmuskels selbst bedingt war, pulsirt der Ventrikel noch lange nach Ausführung der Ligatur fort. Fuhrt man den Versuch mit Pelletierin aus, so tritt nach einigen Minuten wieder Stillstand des Ventrikels ein, woraus ersichtlich, dass das Pelletierin durch seine atropinartige Wirkung den Muscarinstillstand aufgehoben hat. 
Die Wirkung des Pelletierins auf den Frosch lässt sich demnach in Folgendem zusammenfassen:

1. Es findet eine Steigerung der Reflexerregbarkeit des Rïckenmarkes statt, welche bis zu tetanischen Anfällen führt. Anfangs beobachtet man eine ganz geringe Lähmung des Gehirns.

2. Der Mnskel wird wie durch Veratrin, nur in viel geringerem Grade verändert.

3. Es tritt Lähmung der Endigungen des Vagus ein.

\section{Wirkung des Pelletierins auf warmblütige Thiere.}

Das Pelletierin gehört zu den weniger heftig wirkenden Giften. Für Kaninchen war $0,30 \mathrm{~g}$ schwefelsaures Pelletierin die letale Dose, aber auch nur, wenn das Gift direct in die Jugularis injicirt wurde. Selbst subentan werden erbeblich grössere Mengen vertragen, indem offenbar die Ausscheidung des Pelletierins sehr rasch erfolgt. Fir Tauben bildete $0,28 \mathrm{~g}$, für Meersehweinchen $0,25-0,28 \mathrm{~g}$ pro Kilo die tôdtliche Gabe.

Auch bei Warmblittern tritt die Wirkung des Pelletierins auf das Centralnervensystem durchaus in den Vordergrund des Vergiftungsbildes. Erhöhung der Reflexerregbarkeit des Rückenmarkes und Störungen der Locomotion, die wohl auf das Kleinhirn zu beziehen sind, bilden die wichtigsten Vergiftungssymptome, die besonders an Tauben sehr charakteristisch ausgeprägt sind.

\section{Versuch.}

$4 \mathrm{~h} 38 \mathrm{~m}$. Einer Taube von $280 \mathrm{~g}$ Körpergewicht werden $0,05 \mathrm{~g}$ schwefelsaures Pelletierin subcutan injicirt.

42-47 m. Wiederholt heftiges Erbrechen.

$48 \mathrm{~m}$. Es macht sich bei der Bewegung der Beine etwas Ataxie bemerkbar. Das zum Schritt erhobene Bein weicht nach hinten in die Strecklage aus.

$49 \mathrm{~m}$. Erbrechen.

$50 \mathrm{~m}$. Erbrechen.

$53 \mathrm{~m}$. Das Thier fällt mit nach hinten gestreckten Beinen hin. Durch Schlagen der Flitgel arbeitet es sich weiter, wobei die Beine auf dem Boden nachschleifen.

$5 \mathrm{~h} \quad 2 \mathrm{~m}$. Richtet sich mit Mühe auf. Krampf haftes Flügelschlagen. Fällt wieder in die Bauchlage.

$4 \mathrm{~m}$. Kann wieder auf den Beinen stehen, aber unsicher.

$5 \mathrm{~m}$. Erbrechen.

$7 \mathrm{~m}$. Der Kopf wird an den Rücken gezogen.

$14 \mathrm{~m}$. Der in den Nacken gezogene Kopf wird rotirend bewegt.

$19 \mathrm{~m}$. Fällt hin. Bewegt sich, anf dem Bauche liegend, durch Flügelschlagen vorwärts. 
5 h $20 \mathrm{~m}$. Steht wieder. Der Kopf ist in den Nacken gezogen, so dass der Schnabel mit dem Rücken einen Winkel von $90^{\circ}$ bildet. Augen halb geschlossen. Rotationsbewegung des Kopfes. Der Zustand bleibt derselbe, bis

$28 \mathrm{~m}$. der Kopf für einige Secunden in die normale Stellnng gebracht wird, dann aber wieder in die Rückenlage geräth.

$38 \mathrm{~m}$. Die Zeiten, während welcher der Kopf in normaler Lage gehalten wird, nehmen zu. Der wieder in den Nacken gezogene Kopf wird pendelartig bewegt.

$48 \mathrm{~m}$. Einige Brechbewegungen.

$57 \mathrm{~m}$. Der Kopf noch immer rückwärts gezogen, aber nicht mehr so stark. Schnabel und Rücken bilden einen Winkel von ca. $135^{0}$.

$6 \mathrm{~h} 12 \mathrm{~m}$. Symptome lassen nach. Der Kopf fast normal gehalten. Die Beinbewegung wird schon ziemlich beherrscht, so dass das Thier, wenn auch ungeschickt, gehen kann.

$20 \mathrm{~m}$. Alles ziemlich normal. Am anderen Tage ist das Thier ganz normal.

\section{Versuch.}

$11 \mathrm{~h} 27 \mathrm{~m}$. Einer T a ube von $305 \mathrm{~g}$ Körpergewicht werden $0,05 \mathrm{~g}$ schwefelsaures Pelletierin subcutan injicirt.

34-40 m. Wiederholtes Erbrechen unter krampfhaftem Zittern des Körpers.

$44 \mathrm{~m}$. Die Beine gehorchen nicht mehr in normaler Weise dem Willen.

$45 \mathrm{~m}$. Erbrechen.

$50 \mathrm{~m}$. Die Bewegung wird stärker ataktisch.

$58 \mathrm{~m} .0,03 \mathrm{~g}$ schwefelsaures Pelletierin subcutan.

$12 \mathrm{~h} 1 \mathrm{~m}$. Krampfhafte Erschütterung des ganzen Thieres. Es fällt auf den Bauch mit nach hinten gestreckten Beinen. Die Flügel folgen gut dem Willen.

$15 \mathrm{~m}$. Bewegt sich durch Flügelschlagen vorwärts, wobei der Körper auf dem Boden schleift.

$18 \mathrm{~m}$. Kann den Kopf nicht mehr in normaler Stellung halten. Er sinkt zu Boden.

$20 \mathrm{~m}$. Die Athmung beginnt dyspnoisch zu werden.

$23 \mathrm{~m}$. Eine Reihe krampf hafter Erschütterungen.

$25 \mathrm{~m}$. Fast rythmisches Zusammenzucken des ganzen Thieres.

$30 \mathrm{~m}$. Schwache Zuckung der Flügel allein. Respirationsstillstand. Tod.

\section{Versuch.}

$11 \mathrm{~h} 35 \mathrm{~m}$. Einer Ta ub von $275 \mathrm{~g}$ Körpergewicht werden $0,05 \mathrm{~g}$ schwefelsaures Pelletierin subcutan injicirt.

$37-50 \mathrm{~m}$. Wiederholtes Erbrechen.

$53 \mathrm{~m}$. Es beginnt sich Ataxie in den Bewegungen der Beine geltend zu machen. Beim Heben des Beines gelangt dasselbe 
nicht nach vorn, sondern steif nach hinten, bleibt so, wodurch das Thier seitwärts fällt. Bleibt auf dem Bauch mit nach hinten gestreckten Beinen liegen.

$11 \mathrm{~h} 58 \mathrm{~m} .0,03 \mathrm{~g}$ Pelletier. sulfuric. subcutan.

$12 \mathrm{~h} 6 \mathrm{~m}$. Bewegt sich, auf dem Bauch liegend durch Flügelschlagen vorwärts.

13 m. Liegt anf dem Bauch. Kann den Kopf nicht mehr halten.

$19 \mathrm{~m}$. Dyspnoisches Athmen. Zuckungen der Flügel.

$23 \mathrm{~m}$. Respirationsstillstand. Tod.

\section{Versuch.}

$3 \mathrm{~h} 28 \mathrm{~m}$. Einem Meerschweinchen von $406 \mathrm{~g}$ Körpergewicht wird $0,1 \mathrm{~g}$ schwefelsaures Pelletierin subcutan injicirt.

$34 \mathrm{~m}$. Reflexerregbarkeit etwas gesteigert.

$37 \mathrm{~m}$. Beim Gehen geräth das Thier in eine zitternde Bewegung.

$42 \mathrm{~m}$. Die Vorderbeine werden gut dirigirt. Die Hinterbeine gleiten nach auswärts ans. Die Reflexerregbarkeit ist noch mehr gesteigert.

$45 \mathrm{~m}$. Beim Gehversuch gleiten auch die Vorderbeine nach aussen aus, wodurch das Thier auf den Bauch fällt und in eine zitternde Bewegung geräth.

$48 \mathrm{~m}$. Etwas Direction ist auch in den Hinterbeinen, aber sehr wenig. Schiebt sich etwas auf dem Bauche vorwärts.

$54 \mathrm{~m}$. Reflexerregbarkeit hat so weit zugenommen, dass Anblasen Emporschnellen des Thieres bewirkt.

$4 \mathrm{~h} 4 \mathrm{~m}$. Dyspnoisches Athmen.

5 m. Krampfhafte Zuckungen der Extremitäten, besonders der Hinterbeine.

$11 \mathrm{~m}$. Respirationsstillstand. Tod.

\section{Versuch.}

$11 \mathrm{~h} 50 \mathrm{~m}$. Einem Kanin ehen von $1150 \mathrm{~g}$ Körpergewicht werden $0,036 \mathrm{~g}$ schwefelsaures Pelletierin in die Jugularis injicirt.

$52 \mathrm{~m}$. Das Thier kann die Vorderbeine nicht mehr dirigiren, sie gleiten nach auswärts aus.

$54 \mathrm{~m}$. Auch die Direction der Hinterbeine wird mangelhaft.

$12 \mathrm{~h}$ $0,036 \mathrm{~g}$ schwefelsaures Pelletierin.

$2 \mathrm{~m}$. Schiebt sich auf dem Bauche liegend vorwärts.

$5 \mathrm{~m}$. Ab und zu leichtes Zittern des ganzen Thieres. Gesteigerte Reflexerregbarkeit.

$9 \mathrm{~m} .0,072 \mathrm{~g}$ schwefelsaures Pelletierin.

$20 \mathrm{~m}$. $0,072 \mathrm{~g}$ schwefelsaures Pelletierin.

$22 \mathrm{~m}$. Thier liegt auf dem Bauche. Bewegt die Extremitäten, ohne aber von der Stelle zu kommen.

$25 \mathrm{~m}$. $0,072 \mathrm{~g}$ schwefelsaures Pelletierin.

$26 \mathrm{~m}$. Leichte Krämpfe. Der Kopf sinkt in die Seitenlage.

$28 \mathrm{~m}$. Respirationsstillstand. Tod. 
Aus diesen Versuchen ergibt sich, dass auch bei warmblittigen Thieren, ebenso wie beim Frosch, der Ort, an welchem die Wirkung des Pelletierins angreift, das Centralnervensystem ist, und zwar sind es zwei Wirkungen, durch welche das Gift vorherrschend charakterisirt ist :

1. Die Wirkung auf das Ruckenmark, die in einer Erregung: und Steigerung der Reflexerregbarkeit desselben besteht.

2. Die Wirkung auf das Kleinhirn, auf welche die schon bald auftretenden Locomotionsstörungen zu beziehen sind.

Wenden wir uns nun der weiteren Analyse der Wirkungen des Pelletierins za, wie solehe der Versuch am Kymographion gestattet.

10. Versuch. Kaninchen.

\begin{tabular}{|c|c|c|c|c|}
\hline \multicolumn{2}{|c|}{ Zeit } & \multirow{2}{*}{$\begin{array}{l}\text { Druck in } \\
\mathrm{mm} \mathrm{Hg.}\end{array}$} & \multirow{2}{*}{$\begin{array}{l}\text { Pulse in } \\
1 \text { Minute }\end{array}$} & \multirow{2}{*}{ Bemerkungen } \\
\hline h & $\mathbf{m}$ & & & \\
\hline 2 & 20 & $100-108$ & 280 & \\
\hline 2 & 24 & - & - & $\begin{array}{l}0,066 \mathrm{~g} \text { salzsaures Pelletierin in die } \\
\text { Jugularis. }\end{array}$ \\
\hline 2 & 26 & $106-110$ & 270 & Leichte, krampf hafte Zuckung. \\
\hline 2 & 32 & $120-124$ & 240 & \\
\hline$\overline{2}$ & 34 & - & - & $\begin{array}{l}\text { Da die Athmung sehr verlangsamt erscheint, } \\
\text { wird kunstliche Respiration begonnen. }\end{array}$ \\
\hline 2 & 36 & 140 & 250 & \\
\hline 2 & 37 & - & - & Mehrere krampfhafte Zuckungen. \\
\hline 2 & 39 & $130-134$ & 230 & \\
\hline 2 & 40 & $125-128$ & 210 & \\
\hline 2 & 41 & - & - & $\begin{array}{l}0,033 \mathrm{~g} \text { salzsaures Pelletierin. Der In- } \\
\text { jection folgt ein Krampfanfall. }\end{array}$ \\
\hline 2 & 43 & 134 & - & \\
\hline 2 & 47 & - & - & $0,066 \mathrm{~g}$ salzsaures Pelletierin. \\
\hline 2 & 54 & 150 & - & \\
\hline $\mathbf{3}$ & & $150-154$ & 240 & Leichte krampfhafte Zuckungen. \\
\hline 3 & 3 & - & - & $0,033 \mathrm{~g}$ salzsaures Pelletierin. \\
\hline 3 & 6 & $122-126$ & 210 & Beendet. \\
\hline
\end{tabular}

Dieser Versuch zeigte besser wie die vorhergehenden, wie stark das Pelletierin die Reflexerregbarkeit des Riickenmarks erböht. Das nächste Experiment, am curarisirten Thier ausgeführt, sollte entscheiden, ob das Ansteigen des Blutdruckes, wie es Versuch 10 zeigte, in der That als directe Wirkung des Pelletierins oder nur als Folge der Krämpfe aufzufassen war. 
11. Versuch. Kaninchen.

\begin{tabular}{|c|c|c|c|c|}
\hline${ }_{\mathrm{h}}^{\mathrm{Z}}$ & m & $\begin{array}{l}\text { Druck in } \\
\mathrm{mm} \mathrm{Hg} \text {. }\end{array}$ & $\begin{array}{l}\text { Pulse in } \\
10 \text { Sec. }\end{array}$ & Bemerkungen \\
\hline 4 & 38 & $116-120$ & 46 & $\begin{array}{l}\text { Das Thier ist curarisirt worden. Künst- } \\
\text { liche Respiratioń. }\end{array}$ \\
\hline 4 & 39 & $114-119$ & 46 & \\
\hline 4 & 42 & $112-119$ & 46 & $\begin{array}{l}\text { Injection von } 0,03 \mathrm{~g} \text { salzsaurem Pelletie- } \\
\text { rin. Unmittelbar nach der Injection } \\
\text { steigt der Druck auf } 180 \mathrm{~mm} \text {, sinkt } \\
\text { aber dann in einer Minute wieder } \\
\text { anf } 125 \mathrm{~mm} \text {. }\end{array}$ \\
\hline 4 & 45 & $122-126$ & 46 & $\begin{array}{l}\text { Injection von } 0,034 \mathrm{~g} \text { salzsaurem Pelle- } \\
\text { tierin. }\end{array}$ \\
\hline 4 & 48 & $136-142$ & 44 & \\
\hline 4 & 49 & - & - & $\begin{array}{l}\text { Injection von } 0,068 \mathrm{~g} \text { salzsaurem Pelle- } \\
\text { tierin. }\end{array}$ \\
\hline 4 & 52 & 156 & 44 & \\
\hline 4 & 53 & 1 & 4 & \\
\hline 4 & 55 & 160 & 44 & \\
\hline 4 & 57 & 172 & 42 & $\begin{array}{l}\text { Injection von } 0,034 \mathrm{~g} \text { salzsaurem Pelle- } \\
\text { tierin. }\end{array}$ \\
\hline 4 & 59 & 130 & 42 & \\
\hline 5 & & - & - & $\begin{array}{l}\text { Injection von } 0,034 \mathrm{~g} \text { salzsaurem Pelle- } \\
\text { tierin. }\end{array}$ \\
\hline 5 & 2 & 118 & 43 & $\begin{array}{l}\text { Injection von } 0,034 \mathrm{~g} \text { salzsaurem Pelle- } \\
\text { tierin. }\end{array}$ \\
\hline 5 & 7 & 114 & 36 & \\
\hline 5 & 8 & 96 & - & $\begin{array}{l}\text { Erstickung. Der Druck fällt, ohne an- } \\
\text { zusteigen und ohne Grösserwerden der } \\
\text { Pulse, bis auf } 96 \text {, wo aufgehört wird. }\end{array}$ \\
\hline 5 & $91 / 2$ & - & - & $\begin{array}{l}\text { Injection von } 0,034 \mathrm{~g} \text { salzsaurem Pelle- } \\
\text { tierin. }\end{array}$ \\
\hline 5 & 11 & 124 & 42 & \\
\hline 5 & 12 & - & - & $\begin{array}{l}\text { Injection von } 0,034 \mathrm{~g} \text { salzsaurem Pelle- } \\
\text { tierin. }\end{array}$ \\
\hline 5 & 13 & 108 & 38 & \\
\hline 5 & 14 & 70 & 38 & $\begin{array}{l}\text { Erstickung. Der Druck fällt, ohne an- } \\
\text { zusteigen und unter Kleinwerden der } \\
\text { Pulse, bis auf 70, wo anfgehört wird. }\end{array}$ \\
\hline $\begin{array}{l}5 \\
5\end{array}$ & 18 & 118 & 38 & Vagi durchschnitten, gereizt Ohne Erfolo. \\
\hline 5 & $\begin{array}{l}20 \\
28\end{array}$ & $116-122$ & 40 & vagl aurciscninten, gereizt. Unne Erfolg. \\
\hline 5 & 29 & - & - & $\begin{array}{l}\text { Injection von } 0,068 \mathrm{~g} \text { salzsaurem Pelle- } \\
\text { tierin. }\end{array}$ \\
\hline 5 & 30 & - & - & $\begin{array}{l}\text { Injection von } 0,068 \mathrm{~g} \text { salzsaurem Pelle- } \\
\text { tierin. }\end{array}$ \\
\hline 5 & 32 & 100 & - & Versuch beendet. \\
\hline
\end{tabular}


Aus diesem Versuch folgt, dass das Pelletierin den Blutdruck recht energisch steigert und ist die Ursache davon in einer Erregung des vasomotorischen Centrums zu suchen, denn an Thieren, an welchen vorher das vasomotorische Centrum durch Chloral gelähmt worden ist, bleibt die Drucksteigerung ganz aus. Die den arteriellen Druck steigernde Wirkung des Pelletierins darf also nicht, wie bei Digitalin und Physostigmin, auf eine Beeinflussung des Herzmuskels selbst bezogen werden, da bekanntlich letztere Gifte auch nach Lähmung des Gefässnervencentrums ihre Wirkung auf den Druck ausiuben.

Auffallend ist bei der Wirkung des Pelletierin nur, dass die ersten Gaben allein Druckvermebrung hervorrufen, während die späteren so gut wie ganz indifferent sind. Eine Lähmung des Gefässnervencentrums tritt auch durch sehr grosse Gaben von Pelletierin nicht ein. Seine Wirkung auf dasselbe wird wohl so aufzufassen sein, dass es das betreffende Centrum nur vorübergehend reizt, später auf dasselbe aber ohne Einfluss ist.

Ausser der drucksteigernden Wirkung zeigt der Versuch ferner, dass durch Pelletierin eine Lähmung des Vagus eintritt, denn die Reizung desselben ist ohne Erfolg. In Versuchen, die ich nicht im Detail auffuhren will, habe ich dies stets beobachtet und steht dieses Verhalten in guter Uebereinstimmung mit der am Frosch beobachteten atropinartigen Wirkung des Pelletierin.

$\mathrm{Zu}$ den oben erwähnten Wirkungen des Pelletierins auf das Rückenmark und das Kleinhirn treten also noch eine des Gefässnervencentrum erregende und eine den Vagus lähmende hinzu.

Was die Wirkung des Pelletierins auf den Menschen anbetrifft, so liegen darüber in der Klinik von Dujardin-Beaumetz angestellte Versuche vor, die von Rochemure ${ }^{1}$ ) ausführlich mitgetheilt werden. Dieselben sind sowohl mit dem Gemenge der vier Granatwurzelalkaloide, welches anfangs schlechthin Pelletierin genannt wurde, als auch mit dem reinen Pelletierin und Isopelletierin ausgeführt worden. Die Wirkungsweise war in beiden Fällen die gleiche, nur fanden quantitative Unterschiede im Grade der Wirkung statt. Es wurde das Gift subeutan und per os den Versuchspersonen verabfolgt. Locale Affectionen traten nach der subcutanen lnjection nicht auf.

Die Wirkung des Pelletierins ist auch hier wesentlich centraler Natur. Schon nach 0,04 g subcutan stellte sich Schwere des Kopfes

1) Thèse. Paris. 1879. 
ein. Nach $0,1 \mathrm{~g}$ kamen hierzn ein leichtes Schwindelgeftihl, Sehwäche in den Gliedern und Nebelsehen. Die Dosis wurde bis auf $0,5 \mathrm{~g}$ gesteigert, wodurch alle Symptome stärker ausgeprägt wurden. Weiter durfte der sehr starken Schwindelempfindung wegen nicht gegangen werden. Die Wirkung trat meist schon nach 6-8 Minuten ein. In Bezug auf Puls und Temperatur wurde keine Aenderung constatirt. Nach 0,5 g, per os eingeführt, traten dieselben Symptome, nur später, nach $1 / 2-1$ Stunde auf. Das Bewusstsein war immer völlig klar. Diese Versuche wurden an normalen Menschen obne die Absicht einer Bandwurmcur gemacht.

Aus den 26 Krankengeschichten, welche R o che mure (l. c.) mittheilt, und in denen meist $0,4-0,5 \mathrm{~g}$ schwefelsaures Pelletierin gegeben wurde, ist ebenfalls ersichtlich, dass die Vergiftungssymptome in der Regel schon in der ersten halben Stunde auftraten und in Schwindelgefühl, Nebelsehen, Nausea und grossem Schwächegeftihl in den Extremitäten, besonders den Beinen, bestanden. Letzteres wurde häufig so stark, dass Gehen nur mit äusserster Anstrengung möglich war. Ferner traten Gefühl von Ameisenkriechen und manches Mal Wadenkrämpfe auf.

Einzelne Patienten verglichen den Zustand mit Betrunkenheit, wohl des starken Schwindels und der gestörten Locomotion wegen, denn das Sensorium war immer frei. Nach $0,5 \mathrm{~g}$ pflegten die Vergiftungserscheinungen 2-3 Stunden anzuhalten, um dann allmählich sich zu verlieren.

Die am Menschen auftretende Wirkung des Pelletierins lässt sich also dahin zusammenfassen, dass beobachtet wurden:

1. Schwindelempfindung.

2. Sehstörung - NebeIsehen.

3. Schwächegefuhl in den Extremitäten, besonders in den Beinen.

4. Nicht constant waren Uebelkeit, Erbrechen, Zuckungen und Krämpfe einzelner Muskelgruppen, besonders der Wadenmuskeln.

Welche Forderung ist nun an ein gutes Anthelminthicum zu stellen? Dasselbe muss für den Wirth relativ ungefährlich sein, für den Parasiten dagegen deletär. Ferner muss es mit den Abfuhrmitteln die Eigenschaft theilen, nicht schon im Magen resorbirt zu werden, sondern es soll in den Darm gelangen, um bier auf den Parasiten einwirken zu können. Was den ersten Punkt, die Ungefährlichkeit für den Wirth betrifft, so haben die obigen Versuche und Angaben hinreichend dargethan; dass das Pelletierin zu den verhältnissmässig weniger heftig auf den Warmblïter einwirkenden Giften gehört. 
Die toxische Wirkung des Pelletierins auf Bandwirmer anlangend, so konnten dartiber natïrlich nur therapeutische Versuche entscheiden und haben es ja auch, wie eingangs erwähnt, bereits gethan. Doch bevor ich auf dieselben eingehe, ist es von Interesse, zu fragen, ob sich nicht im physiologisehen Versuch an isolirten Bandwürmern die Wirkung des Pelletierins auf dieselben darthun lässt und in welchem Grade es sich hierbei giftig erweist. Leider stehen zu dergleichen Versuchen die im Mensehen vorkommenden Bandwürmer wobl nur äusserst selten, wenigstens in normalem Zustande, zur Disposition. Ich habe daher die Wirkung des Pelletierins auf den Bandwurm der Katze, die Taenia serrata, untersucht. Die Entozoen wurden dem Darm der eben getödteten Thiere entnommen und sofort in eine auf $37^{\circ}$ erwärmte Lösung, welche 1 Proc. Kochsalz und 0,1 Proc. kohlensaures Natron enthielt, wie dieselbe von $\mathrm{Bunge}{ }^{1}$ ) für Ascariden angegeben worden ist, gesetzt. In derartigen, auf Körpertemperatur erwärmten Salzlösungen leben die Taenien mehrere Tage, bewegen sich und scheinen sich normal zu verhalten. Setzt man einer solchen, eine Taenia enthaltenden Lösung Pelletierin im Verhältniss von 1:10000 zu, so hören schon nach 5 Minuten die Bewegungen des Thieres auf. Bringt man dasselbe nun in frische, erwärmte Salzlösung, so pflegen nach 15-30 Minuten die Bewegungen sich wieder einzustellen. Wirkte die Giftlösung länger wie 10 Minuten auf die Taenie ein, so fand Wiederherstellung nicht statt.

Wenden wir uns jetzt zur Betrachtung der therapeutisehen Resultate, welche durch Anwendung der Pelletierine bei Bandwurmeuren erzielt worden sind. Die grösste Anzahl von Fällen ist in der Klinik von Durjardin-Beaumetz beobachtet worden, der gleich nach Entdeckung der Granatalkaloide Versuche mit denselben anstellen liess. Doch berichtet Roch e mu re auch über die von anderen Pariser Aerzten erlangten Erfolge. Die Vorcur wurde meist so ausgefiihrt, dass der Patient am Abend vorher eine leichte Nahrung (Milch u. s. w.) zu sich nahm und dann ein gelindes Abführmittel erhielt. Am anderen Morgen wurde die Pelletierinlösung nuichtern genommen und nach $1 / 2-2$ Stunden ein energisches Abfiuhrmittel, Jalapentinctur, Ricinusöl oder Sennesblätterinfus gegeben.

In die erste Reihe werden wir diejenigen Fülle rechnen mïssen, in denen das Gemenge der schwefelsauren Salze der vier Alkaloide benutzt wurde. Die Dosis betrug $0,4-0,5 \mathrm{~g}$, die in $30 \mathrm{~g}$ Wasser

1) Zeitschr. f. physiol. Chem. Ba. 8. S. 48. 
gelöst gegeben wurden. Es handelte sich fast ausschliesslich um Taenia inermis.

$$
\text { Anzahl der Fälle }=26 \text {. }
$$

In 8 Fällen erfolgte Abgang des Parasiten mit Kopf.

In 8 Fällen fehlte nur der Kopf.

8 mal war das Thier einschliesslich des verengten Theiles da, aber Halspartie und Kopf fehlten.

2 mal erfolgte nur Abgang von Theilen des Parasiten.

Wenn oben unter den an ein gutes Anthelminthicum zu stellenden Anforderungen auch die aufgeführt wurde, dass dasselbe schwer resorbirbar sein mïsse, so entsprechen die schwefelsauren Salze der Pelletierine derselben nicht. Dieselben sind in Wasser sebr leicht löslich und es muss vermuthet werden, dass von ihnen schon ein beträchtlicher Theil im Magen resorbirt wird und so dem therapeutisehen Zweck verloren geht. In der richtigen Ueberlegung, dass die Wirkung der Pelletierine den Parasiten energischer treffen würde, wenn man die Resorption derselben im Magen möglichst zu verhindern suchte, änderte man nun die obige Ordination dahin, dass zu den $0,4-0,5 \mathrm{~g}$ der schwefelsauren Salze $0,5 \mathrm{~g}$ Gerbsäure zugesetzt wurde. Wie mit den meisten Alkaloiden bildet nämlich die Gerbsäure auch mit den Pelletierinen schwer lösliche Verbindungen. Das Resultat entsprach völlig der Voraussetzung, denn von

33 Fällen trat in

$29=$ Abgang des Parasiten mit Kopf ein.

Mittlerweile war es Tanret gelungen, die einzelnen Alkaloide zu isoliren. Bei der nun möglich gewordenęn Untersuchung der einzelnen Alkaloide auf ihre Wirkung zeigte sich, wie eingangs bereits bemerkt, dass dem Methyl- und Pseudopelletierin, in den angewandten Dosen wenigstens, keine anthelminthische Wirkung zukommt. Berenger-Feraud ${ }^{1}$ ) erzielte in 31 Fällen, in denen er Methylpelletierin, Pseudopelletierin, sowie ein Gemenge beider benutzte, keinen einzigen Erfolg.

Sebr günstig dagegen stellten sich die Resultate bei Anwendung. eines Gemenges von Pelletierin und Isopelletierin, wenn dieselben mit Gerbsäure gegeben wurden.

Berenger-Feraud erhielt in 30 Fällen 26 Erfolge,

Dujardin-Beaumetz ${ }^{2}==39=37=$

Laboulbène ${ }^{3}$ ) $\quad=19=19=$

wo unter Erfolg Abgang des Parasiten mit Kopf zu verstehen ist.
1) Bullet. de thérap. 1879 .
2) Bullet. de thérap. Bd. 98. p. 433.

3) Bei Dujardin-Beaumetz. 
Bei Anwendung eines Gemenges der schwefelsauren Salze des Pelletierins und Isopelletierins ist $0,3-0,4 \mathrm{~g}$ eine völlig gentlgende Dosis. Gewöbnlich wurden $0,3 \mathrm{~g}$ verwandt und nach einer halben Stunde bereits das Abftihrmittel gegeben.

Aus der deutschen Literatur ist mir nur eine Publication über die therapeutische Wirkung des Pelletierins bekannt, von Friedmann ${ }^{1}$, welcher das Gemenge der schwefelsauren Salze mit der dreifachen Menge Gerbsäure in 10 Fällen mit Erfolg anwandte und und uber das Mittel sehr günstig urtheilt. Die von Friedmann benutzten Dosen waren erheblich grösser, denn er liess nach gewöhnlicher Vorbereitungscur alle halbe Stunde $0,5 \mathrm{~g}$ (1 Theil schwefelsaures Pelletierin auf 3 Theile Gerbsäure) nehmen. Es waren 3 bis $6 \mathrm{~g}$ erforderlich, also entsprechend $0,75-1,5 \mathrm{~g}$ der schwefelsauren Salze. Die Herkunft des von ihm benutzten Präparates ist nicht angegeben.

Vergleichen wir nun die oben vom Pelletierin für eine erfolgreiche Bandwurmeur als ausreichend angegebene Dose $(0,3 \mathrm{~g}) \mathrm{mit}$ den Mengen, welche im physiologischen Versuch als für die Taenia letal festgestellt wurden, und fragen wir, ob hier gute Uebereinstimmung vorhanden ist, so werden wir, vorausgesetzt, dass für Taenia inermis dieselben Concentrationen des Giftes tödtlich sind wie für Taenia serrata, die Frage bejahen missen. Selbst wenn von den $0,3 \mathrm{~g}$ der dritte Theil im Magen bereits resorbirt würde, was nicht wahrscheinlich ist, so werden doch im Darm eines nulchternen $\mathbf{P a}$ tienten Concentrationen zu Stande kommen, die 1:10000 weit übertreffen. Rechnen wir z. B. die im Darm in Frage kommende Flitssigkeitsmenge auf etwa $200 \mathrm{cc}$, was wohl zu hoch ist, so wurde sich der Gehalt an Pelletierin noch immer wie $1: 1000$ (0,2:200) stellen, also durchaus zur Tödtung des Parasiten ausreichen miissen. - Aus den physiologischen Versuchen werden wir eine Contraindication für die Anwendung des Pelletierins ableiten müssen. Da, wie oben gezeigt, eine, wenn auch nicht andauernde, so doch recht beträchtliche Steigerung des arteriellen Blutdruckes durch dasselbe stattfinden kann, so wird von seiner Benutzung da Abstand zu nehmen sein, wo Aneurysmen, atheromatös entartete Gefässe vorliegen, kurz in allen den Fällen, wo eine Steigerung des arteriellen Blutdruckes Gefahr bringen kann.

Neuerdings ist von Kamnitzer eine Arbeit: „Ueber die Wirkungsweise der Granatwurzelrinde " 2), erschienen, in welcher derselbe die

1) Centralbl. f. d. ges. Therapie. 1884. Heft 3. S. 100.

2) J. Kam nitzer, Dissert. Berlin 1883. 
Ansicht vertritt, dass bei den mit dem Decoct der Granatwurzelrinde ausgefthrten Bandwurmeuren die anthelminthische Wirkung nicht auf das Pelletierin zurtickgeführt werden könne, da letzteres seiner Flichtigkeit wegen bei Anfertigung eines Decoctes oder Macerationsdecoctes völlig verschwunden sein müsse, und schreibt er die anthelminthische Wirkung derartiger Decocte wesentlich der Gerbsäure zu. Ebenso siebt er die bei Bandwurmcuren mit Cortex radicis Granati beobachteten Vergiftungserscheinungen nur als durch die Gerbsäure hervorgerufen an.

Was den ersten Punkt, das Entweichen des Pelletierins aus den Decocten, betrifft, so fuhrt Kamnitzer keine Versuche zur Begründung seiner Ansicht an. Es ist aber a priori wenig wahrscheinlich, dass aus einem Rindendecoct, welches so stark saner wie das von Cortex Granati ist, eine starke Verflüchtigung der Pelletierine eintreten werde. Von der Richtigkeit dieser Vermuthung habe ich mich durch Versuche iberzeugt, in denen $50 \mathrm{~g}$ Granatwurzelrinde mehrere Stunden mit 300 ec Wasser in einer Retorte mit Kuhler erhitzt und die ersten übergegangenen 100 ce Destillat aufgefangen wurden. Das neutral reagirende Destillat wurde nun mit Kalkhydrat versetzt und wieder destillirt. Jetzt ging eine Base tuber und wurde die Procedur bis zum Auf hören der alkalischen Reaction fortgesetzt. Die tibergegangene Base, deren Menge durch Neutralisiren mit titrirter Schwefelsäure auf weniger als $3 \mathrm{mg}$ (auf Ammoniak berechnet) geschätzt wurde, bestand nur aus Ammoniak. Um zu untersuchen; ob die benutzten Granatwurzelrinden auch hinreichend Pelletierine enthalten hatten, wurde das Decoct nach Abdestillirung der 100 cc mit Kalkmilch versetzt und bis zum Aufhören der alkalischen Reaction destillirt. Jetzt fand reichlicher Uebergang von Pelletierinen statt, die in ublicher Weise vom Ammoniak getrennt, als salzsaure Salze ca. $0,3 \mathrm{~g}$ wogen.

$\mathrm{Da}$ bekanntlich frische Granatwurzelrinde viel sicherer wirkt wie alte, so suchte man nach Tanret's Entdeckung diesen Umstand daraus zu erklären, dass beim Liegen der Rinde das Pelletierin sich verflïchtigt und dieselbe dadurch unwirksam wird. Diese Annahme wird wohl kaum richtig sein, denn wenn beim Anfertigen von Decocten das Pelletierin mit den Wasserdämpfen nicht entweicht, wird wohl noch weniger eine Verflüchtigung bei längerem Aufbewahren der Rinde stattfinden. Wahrscheinlicher erscheint es, dass beim Liegen der Rinde eine Zersetzung der Alkaloide eintritt, wohl durch Oxydation, da dieselben bekanntlich leicht an der Luft durch Sauerstoffaufnahme verharzen. - Der Umstand aber, dass alte Rinde so häufig wirkungslos ist, spricht auch gegen die Ansicht, dass vorherr- 
schend die Gerbsäure der antbelminthiseh wirkende Bestandtheil ist, denn die Rinden bïssen durch Aufbewahren an ihrem Gerbsäurereichthum nur verhältnissmässig wenig ein.

Was ferner die Anschauung $\mathrm{K}$ amnitzer's anlangt, dass die Vergiftungssymptome, wie dieselben nach Einnabme des Decoctes der Granatwurzelrinde beobachtet werden, nur Folgen der Gerbsäure sind und keinerlei Erscheinungen auf eine Pelletierinwirkung schliessen lassen, so kann ich derselben auch nicht beistimmen. Die Störungen, . welche sich nach Einführung des Granatwurzelrindendecoctes im Verdauungskanal geltend machen, Ekel, Erbrechen, Leibschmerzen, sind gewiss zum allergrössten Theil auf die Gerbsäure als Ursache zurtickzuführen. Anders aber verhält es sich mit den Symptomen, welche nach grösseren Gaben (mehr als $60 \mathrm{~g}$ Rinde) sich als Allgemeinvergiftung geltend machen, denn dann „ entstehen Eingenommenheit des Kopfes, Schwindel, Schläfrigkeit, undeutliches Sehen, Einschlafen der Glieder und in manchen Fällen krampfhafte Zuckungen, namenthich der Wadenmuskeln. "1) Dies aber sind gerade Symptome, von denen wir oben gesẹhen haben, dass sie für das Pelletierin charakteristisch sind.

Ein wesentliches Hinderniss, warum das Pelletierin in Deutschland verhältnissmässig noch selten zur Bandwurmeur benutzt wird, ist sicherlich in dem hohen Preise desselben zu suchen. 'Die zu einer Cur nöthige Dose, welche von Tanret in Lösung versandt wird, kostet 8 Mark. - Es wäre nun in solchen Fällen, in denen dieser Preis ein zu hoher ist und man infolge dessen sich des Granatrindendecoctes bedient, entschieden zweckmässig, wenn es gelänge, letzteres von derjenigen Substanz, welche seinen Geschmack so widerwärtig macht und zu so viel Störungen Veranlassung gibt, zu befreien, dạs heisst, von der Gerbsäure. Das lässt sich nun folgendermaassen erreichen.

Man fertigt das Decoct durch etwa einstundiges Erbitzen der gut zerkleinerten Rinde auf dem Wasserbad an, so dass man sicher sein kann, dass alle Alkaloide in Lösung übergeführt worden sind. Dann lässt man erkalten und setzt Kalkmilch im Ueberschuss zu. Da hierdurch das Decoct eine fast breiige Consistenz annimmt, so wende man nicht zu wenig Wasser zum Decoct an. Man filtrirt nun die Flïssigkeit vom Niederschlag ab und wäscht gut nach. Das fast wasserhelle, leicht gelblich gefärbte Filtrat reagirt alkalisch. Man neutralisirt es mit Schwefelsâure. In dem Filtrat sind die Alkaloide

1) Nothnagel und Rossbach, Arzneimittellehre. 3. Aufl. S. 771. 
alle vorhanden, während die Gerbsäure ganz entfernt worden ist, da dieselbe als gerbsaurer Kalk auf dem Filter geblieben ist. Es hat infolge dessen die so gewonnene Flïssigkeit keinen adstringirenden Geschmack mehr und kann bei Zusatz von Geschmackscorrigentien wie Limonade getrunken werden. Der vatürlich in der Lösung vorhandene schwefelsaure Kalk darf als für die Wirkung gleichgültig: angesehen werden. Man kann ibn, wenn man will, durch Zusatz von etwas phosphorsaurem Natron und nochmalige Filtration leicht entfernen. Zweckmässig aber wird es sein, eine gewisse Quantität Gerbsäure $(2-3 \mathrm{~g})$ der Flïssigkeit zuzusetzen, um die Resorption des Pelletierins im Magen möglichst zu verhindern. Hat man den Kalkuiberschuss aus dem Filtrat nicht entfernt, so entsteht dadurch ein Niederschlag und wählt man dann die Gerbsäuremenge etwas grösser. Die Schwierigkeit einer derartigen Zubereitung der Granatrinde zum therapeutischen Gebranch wïde hauptsächlich darin liegen, dass die Ordination für den Apotheker zu complicirt ist.

Aus diesem Grunde dürte es wohl entsprechender sein, aus der Granatrinde ein gerbsäurefreies, alle Alkaloide aber enthaltendes Extract herzustellen, was, wie folgt, möglich ist. Das, wie oben angegeben, von Gerbsäure befreite, mit Schwefelsäure genau neutralisirte Filtrat des Decoctes wird auf dem Wasserbad bis fast zur Trockne gebracht und dann mit Alkohol von 70 Proc. versetzt. Der schwefelsaure Kalk bleibt hierbei zurück, während die Pelletierinsalze in die alkoholische Lösung gehen. Der Rückstand des alkoholischen Auszuges stellt dann das Extract dar. Dasselbe enthält ausser den Salzen der Granatalkaloide noch Salze der Alkalien mit organisehen Säuren. Es ist beinahe als ganz krystallinisch zu bezeichnen. In Wasser ist es mit geringer Trübung löslich. Bei den von mir in dieser Weise verarbeiteten Granatwurzelrinden gaben $20 \mathrm{~g}$ ca. $1,0 \mathrm{~g}$ Extract. Wie viel die Rinde der Aeste and des Stammes an derartigem Extract liefert, habe ich noch nicht untersucht. Auch bei Anwendung des Extractes wird ein Zusatz von ca. 1,0-2,0 g Gerbsàure aus oft erwähntem Grunde stattfinden müssen.

Die neue französische Pharmacopoe führt ebenfalls ein Granatwurzelrindenextract auf, welches in der Weise angefertigt wird, dass die halb fein gepulverte Rinde mit Alkohol von 60 Proc. 12 Stunden lang bei gewöhnlicher Temperatur extrahirt wird, dann colirt, der Alkohol abdestillirt und der Rückstand zur Consistenz eines weichen Extractes eingedampft wird. Die Schattenseite desselben ist offenbar darin zu suchen, dass hierbei die Gerbsäure zum grossen Theil mit ausgezogen wird. 
Die Vorzlige, welehe die Benutzung des von mir vorgeschlagenen Extractes aus Cortex Granati vor der Anwendung des Decoctes haben wïrde, sind in Folgendem zu sehen.

1. Es wird die störende Wirkung der grossen Gerbsäuremengen sowie der widerwärtige Geschmack des Decoctes vermieden.

2. Es wird möglich sein, augh solche Rinden zur Cur zu benutzen, welche ihrer relativen Pelletierinarmuth wegen als Decoet wirkungslos sind, die aber brauchbar werden würden, wenn in obiger Weise eine Concentration der wirksamen Bestandtheile unter Ausschluss der Gerbsäure stattfände.

Es werden natürlich die therapeutischen Versuche darüber entscheiden, ob der Erfolg bei Benutzung des Extractes den Erwartungen entspricht. Ferner wird die fabrikmässige Darstellung des Extractes, welche ich an geeigneter Stelle anregen werde, erst zeigen, wie hoch der Preis desselben sich stellen wird, was in unserem Falle natürlich auch von grösster Wichtigkeit ist. 